\title{
Ptychographically-assisted X-ray fluorescence nanotomography for characterization of complex materials
}

\author{
D. Karpov ${ }^{1}$, M. Holler², D. Ferreira Sanchez ${ }^{2}$, A. Diaz ${ }^{2}$ \\ ${ }^{1}$ European Synchrotron Radiation Facility, 71 Avenue des Martyrs, Grenoble, France, \\ ${ }^{2}$ Paul Scherrer Institut, Forschungsstrasse 111, Villigen, Switzerland \\ dmitry.karpov@esrf.fr
}

Information on 3D elemental distribution is critically important in studies of catalysis, life sciences, materials research, toxicology, geology, and many other fields. To access this information X-rays are advantageous compared to electron microscopy and atom probe tomography techniques because of their short wavelengths, high penetrability and possibility to image samples non-destructively. Xray fluorescence (XRF) tomography stands out among other synchrotron-based techniques for its high sensitivity to low elemental concentrations, simultaneous access to many elements in a single measurement, and for its compatibility with other simultaneous imaging modalities. Recent developments of nano-focussing optics for hard X-rays have advanced XRF tomography towards submicron resolution.

Although higher resolution can be easily achieved in 2D, practical limitations due to long scanning times and instrument instability and positioning accuracy have limited the $3 \mathrm{D}$ resolution to a few hundred nanometers $[1,2]$. With expected improvements of brilliance at upgraded diffraction-limited storage rings, this limit will be pushed through the reduction of acquisition time. However, resolving the sample stability and positioning accuracy issue will immediately benefit existing XRF imaging stations (both for 2D and $3 \mathrm{D}$ case) and will have an even greater impact on the imaging quality of upgraded storage rings.

In this talk, we will present current developments in combining XRF tomography and ptychographic X-ray computed tomography (PXCT) at the cSAXS beamline of the Swiss Light Source. In this approach, we use flOMNI (flexible tOMography Nano Imaging, see Figure 1) [3], a unique instrument that allows accurate sample positioning with respect to the focusing optics by means of differential laser interferometry. The setup is now equipped with a fluorescence detector. Overcoming the stability issue gives us an opportunity to achieve resolution in XRF tomography on the level of the probe size with minimal data manipulation. While combining the electron density contrast from PXCT and the element specificity of fluorescence allows us to exercise various data analysis strategies that will be covered in this talk. We expect that this development will find interest from synchrotron users in fields ranging from life sciences to materials science.

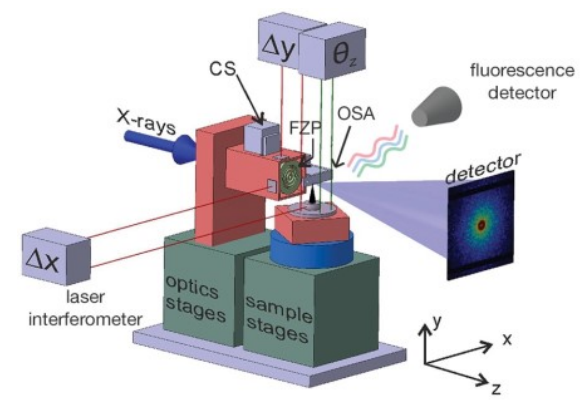

Figure 1. flOMNI (flexible tOMography Nano Imaging) instrument with fluorescence detector. The acronyms used are a central stop (CS), a Fresnel zone plate (FZP) and an order-sorting aperture (OSA). Adapted from ref. [3].

[1] T. Victor et al., X-ray Fluorescence Nanotomography of Single Bacteria with a Sub-15 nm Beam, Sci. Rep. 8, 13415 (2018).

[2] G. Martínez-Criado et al., ID16B: a hard X-ray nanoprobe beamline at the ESRF for nano-analysis, J. Synchrotron Rad. 23, 344-352 (2016).

[3] M. Holler et al., X-ray ptychographic computed tomography at $16 \mathrm{~nm}$ isotropic 3D resolution, Sci. Rep. 4, 3857 (2014).

Keywords: ptychographic x-ray computed tomography; x-ray fluorescence tomography; scattering; spectroscopy 